\title{
CFD MODEL OF A MAGNETORHEOLOGICAL FLUID IN SQUEEZE MODE
}

\author{
Bogdań SAPIŃSKI*, MarcinSZCZĘCH* \\ *AGH University of Science and Technology, Faculty of Mechanical Engineering and Robotics, \\ al. Mickiewicza 30, 30-059 Kraków, Poland \\ deep@agh.edu.pl, szczech@agh.edu.pl
}

\begin{abstract}
The study briefly outlines a CFD model of a magnetorheological (MR) fluid operated in squeeze mode with a constant interface area using the CFD (Computational Fluid Dynamics) approach. The underlying assumption is that the MR fluid is placed between two surfaces of which at least one can be subject to a prescribed displacement or a force input. The widely employed Bingham model, which fails to take into account the yield stress variations depending on the height of the gap, has been modified. Computation data obtained in the ANSYS CFX environment are compared with experimental results.
\end{abstract}

Key words: MR Fluid, Squeeze Mode, CFD Model, Bingham Model

\section{INTRODUCTION}

MRfluids are heterogeneous suspensions of ferromagnetic particles suspended in a carrier liquid (typically oil or water) whose rheological properties can be controlled through the action of a magnetic field. The fluids can be operated in valve mode, in direct shear/clutch mode or in squeeze mode. When operated in squeeze mode, properties are dependent on application requirements. In the case of vibration damping systems, of particular importance is the ability to generate significant forces when MR fluid is displaced from the squeeze zone and to provide for a wide variability range of rheological parameters. Two cases of squeeze mode operation of MR fluids are considered: with the constant volume or with the constant interface area. Stresses experienced by this fluid when squeezed approach 100-150 kPa with displacements of the orderof several millimeters.

MR fluids operated in squeeze mode experience some characteristic effects, one of them being the displacement of the carrier fluid from squeeze zone. As a result, the properties of the fluid will change during its operation in squeeze mode. Another phenomenon involves the change of the fluid structure: in the presence of a magnetic field, ferromagnetic particles arranged in column-like chain structures get deformed due to compression loading. In the consequence, the MR fluid provides increased resistance to compressive loading and its yield stress tends to increase. Another aspect of the fluid behavior in squeeze mode is the "clumping behavior"(Farjoud et al., 2011) which involves aggregation of fluid particles leading to formation of ferromagnetic particle aggregate clumps in the fluid. As a result, progressively increasing force will develop in consecutive test runs.

One has to bear in mind, however, that until recently literature on the subject would abound in reports on the valve and coupling modes whilst the reports on squeeze mode were but a few. In recent years, however, squeeze mode has received a great deal of attention. The modeling of squeezeing force is investigated inFarjoud et al., (2009) and Horak (2013). However, models presented there are most complicated and hardly applicable in engineering design. This study is focused on the CFD model of MR fluid operated in squeeze mode with the constant interface area (Fig 1). It is assumed that fluid is found in between two parallel surfaces. A widely employed Bingham model has been modified as it fails to take into account the yield stress variations depending on the height of the gap. The numerical calculations were performed in the ANSYS CFX environment (ver. 12). Computation data are compared with experimental results, summarised in Sapiński et al., (2013).
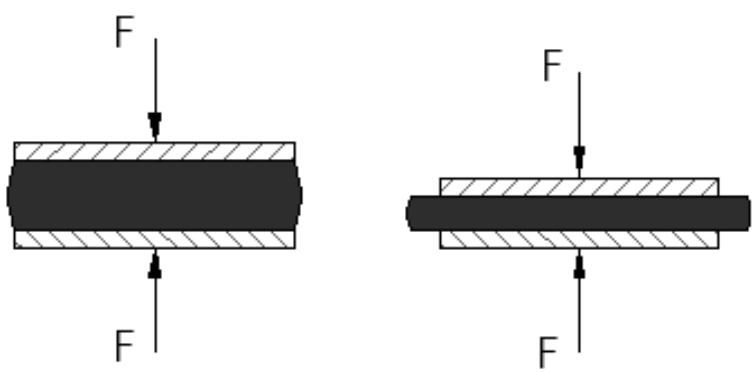

Fig. 1. MR fluid in squeeze mode with the constant interface area

\section{MRFLUID MODEL}

MR fluids are non-Newtonian, shear-thinned fluids. When exposed to the action a magnetic field, the structure of ferromagnetic particles changes, giving rise to stress. The dynamic viscosity and the yield stress will change, too. To capture the variability of these parameters we rely on the model of apparent viscosity of a MR fluid implemented in the ANSYS CFX environment:

$\mu_{\text {app }}=\frac{T_{0}}{\dot{y}}+\mu$

where: $\mu_{\text {app }}$ - apparent viscosity, $\mathrm{T}_{0}$ - yield stress, $\dot{V}$ - shearing rate, $\mu$-dynamic viscosity.

This model has been effectively used in several applications 
and theoretical analyses, including (Gavin, 2001; Mazlan, 2008). When the yield stress (dependent on the magnetic field strength) is exceeded, the MR fluid begins to yield. In squeeze mode, the yield stress is also dependent on the height of the fluid gap (Wang et al., 2011).

\section{EXPERIMENTS}

Squeeze force testing was done in the experimental set-up comprising a rheometer(Salwiński et al., 2013) with a fluid gap, formed by two parallel, flat plates. One of these plates remained immobile whilst the other moved in the progressiveretractivemotion. This configuration is used when testing the properties of magnetic fluids, particularly with the use of Anton Paarrheometers (http://www.anton-paar.com).

MRfluid selected for testing is the MRF-122G manufactured by the Lord Corporation (http://www.lord.com), which is recommended for use in vibration dampers. Its properties are: density: $2.38 \mathrm{~g} / \mathrm{cm} 3$, saturation magnetization: $359 \mathrm{kA} / \mathrm{m}$, volumetric fraction of ferromagnetic particles in the carrier fluid: $22 \%$.

\subsection{Test rig}

The use of a rheometer enables the magnetic fluids to be investigated when exposed to the magnetic field, both in the shear and squeeze mode. The schematic diagram of the test chamber is shown in Fig. 2.

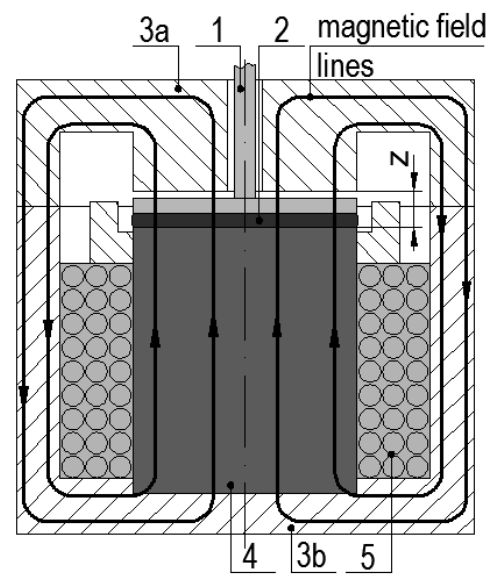

Fig. 2. Schematic diagram of the test chamber: 1-plate, 2-MR fluid, 3a-upper housing, 3b-lower housing, 4-electromagnet core, 5 -coil

In the course of the experiment the height of the fluid gap was varied in accordance with the predetermined function governing the plate position (with the use of a step motor). This plate is an element with non-magnetic properties. The accuracy of the plate position measurement was $0.001 \mathrm{~mm}$. The squeezing force was measured with a force sensor. Experiments were conducted in thermallystabilised conditions, the ambient temperature being $25^{\circ} \mathrm{C}$. Magnetic field was generated by current flowing in the electromagnet windings. The magnetic core and the chamber housing are magnetic circuit components and are made of ferromagnetic materials. Fluid being squeezed was in the lower section of the fluid gap.

The measurement and control system used in the experiments comprised a computer with a card National Instruments USB-6211, supported by the LabView software (version 2009) (www.ni.com). Measurements were taken of the plate position, of velocity of a linear motor, the squeeze force and of temperature inside the test chamber. The measurement and control system was also used to control the intensity of current flowing in the electromagnet coil and the drive of the step motor.

The cross-section of the fluid gap is shown in Fig. 3. The mobile plate was placed between the electromagnet core and the housing with the diameter $d=45 \mathrm{~mm}$. The gap height $z=10 \mathrm{~mm}$ between the core and the chamber's upper lid allows an unconstrained movement of the plate. The volume of the tested fluid sample was $1.6 \mathrm{ml}$ and the initial plate height was $h=1 \mathrm{~mm}$. The plate's position was varied by $\Delta h=0.2 \mathrm{~mm}$ and the squeezing time was $t=5 \mathrm{~s}$. This rheometer configuration ensures an almost uniform magnetic field distribution in the gap.The analysis of the magnetic field distribution in the rheometer used in tests is presented elsewhere (Sapiński et al., 2013).

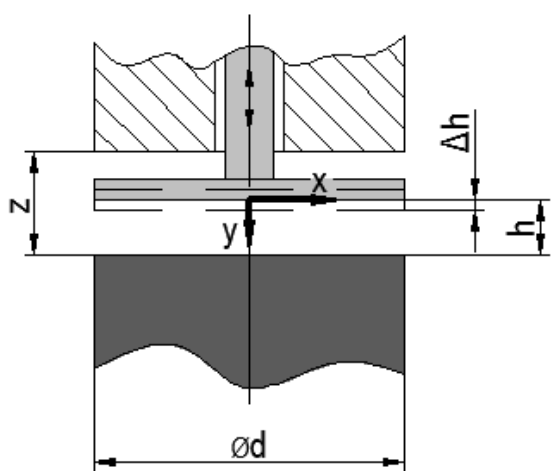

Fig. 3.Gap cross-section in the test chamber

\subsection{Results}

Experiments were performed for the maximal gap height, under the magnetic field with the flux density B: $70,100,140,200,270 \mathrm{mT}$.

The plot of squeezing force in the function of time is shown Fig. 4, for the given initial values of magnetic flux density. The plate's position during the experiments is indicated with the dotted line. The curves obtained for the first two applied excitations $B$ are nearly linear in shape. In the remaining cases $(140,200,270 \mathrm{mT})$, it appears that the force would increase at a faster rate for $\Delta \mathrm{h}$ from the range $(0 \div 0.03$ $\mathrm{mm}$ ) than $(0.03 \div 0.2 \mathrm{~mm})$.

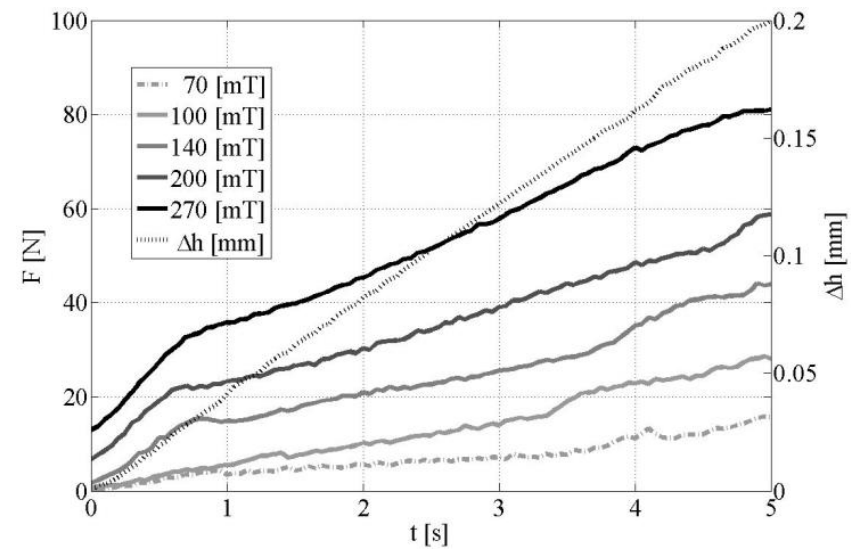

Fig. 4. Squeezing force $\mathrm{F}$ and position $\Delta \mathrm{h}$ vs time 
For the flux density range $(100 \div 270 \mathrm{mT})$ and for the initial plate position (i.e. for the maximal gap height), a nonzero normal force was registered (Fig. 5). It is the result of pressure generated in the MR fluid under the action of magnetic field, and its value is obtained from the formula (Rosenweig, 1985):

$\mathrm{p}=\mu_{0} \mathrm{MH}$

where: $\mathrm{p}$ - pressure, $\mu_{0}$ - magnetic permeability in the vacuum, $\mathrm{M}$ - magnetisation, $\mathrm{H}$ - magnetic field strength.

A detailed analysis of this phenomenon is provided in the work (Salwiński and Horak, 2012). This pressure is not considered in rheological models of MRfluids, including the Bingham model adopted by the author.

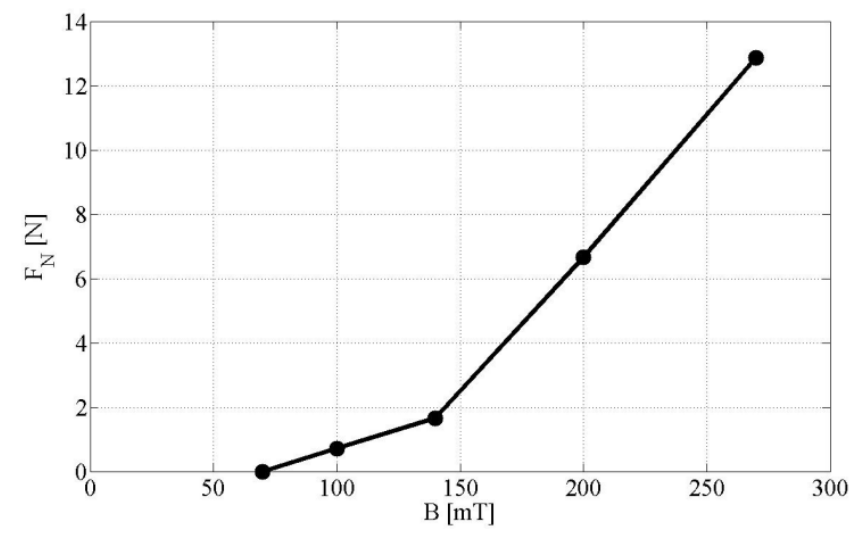

Fig. 5.Normal force $F_{N}$ vs magnetic flux density $B$

\section{NUMERICAL CALCULATIONS}

In the adopted configuration, the distributions of velocity and MR fluid pressures registered in squeeze mode will vary. For that very reason, the squeezing force is not easily defined on the basis of mathematical equations and the numerical procedure was applied instead, using the ANSYS CFX environment (http://www.lord.com). This is a dedicated program for modeling the flows of fluids, gases, mixtures and chemical reactions. The calculation procedure is based on the finite volume method (FVM).

The case considered in this study is axi-symmetrical. To reduce the number of finite elements and to shorten the calculations, the procedure was applied to $1 / 12$ th of the entire model $(150)$. The symmetry conditions were defined on the edges of the cut-out area. As the rate of changes of the plate's position was rather small, the numerical procedure was restricted to the laminar flow model as no turbulent flows were encountered (Horak, 2013). The finite elements in the modeled system are pyramids with the average height $0.1 \mathrm{~mm}$. The numerical simulations neglect the thermal processes in the system (an isothermal system). As the height of the fluid in the gap is rather small $(\mathrm{h}=1[\mathrm{~mm}])$, the effects of gravity are neglected. No slip wall boundary conditions were imposed on the upper and lower surfaces, respectively.

Underpinning the CFD simulations is the Bingham model. The plot of measured squeeze forces for $B=100 \mathrm{mT}$ is shown in Fig. 6, providing also the numerical data obtained for the constant value of yield stress $\mathrm{T}_{0}=10000 \mathrm{~Pa}$ in the Bingham model. This value was assumed basing on the MR fluid manufacturer's specifications (http://www.lord.com).

The squeezing force determined by the CFD calculations was about $10 \mathrm{~N}$ and it increased slightly with the elevation of the plate's position. The differences between the calculation and measurement data are significant for an MR fluid in squeeze mode with the constant yield stress. Thus determined squeeze force fails to capture the real behavior of fluid.

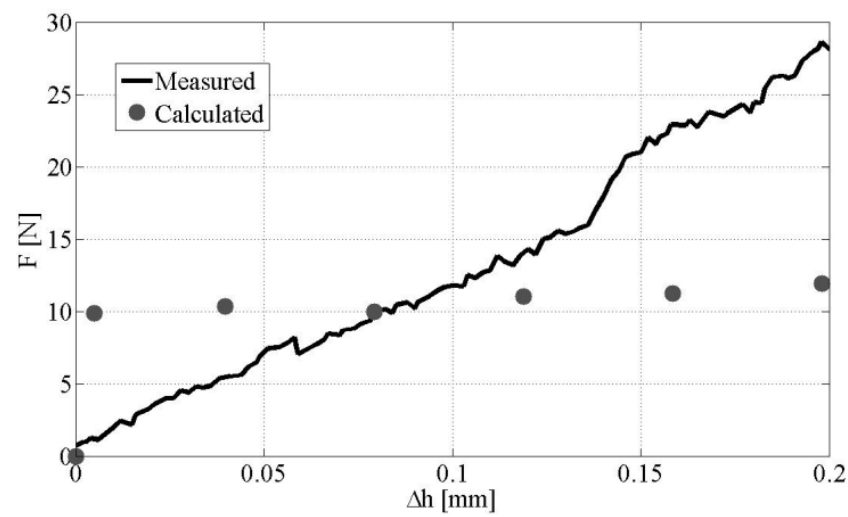

Fig. 6. Squzeeing force $F$ vs position $\Delta h$; magnetic flux density $B=100 \mathrm{mT}$, yield stress $\mathrm{T}_{0}=$ const

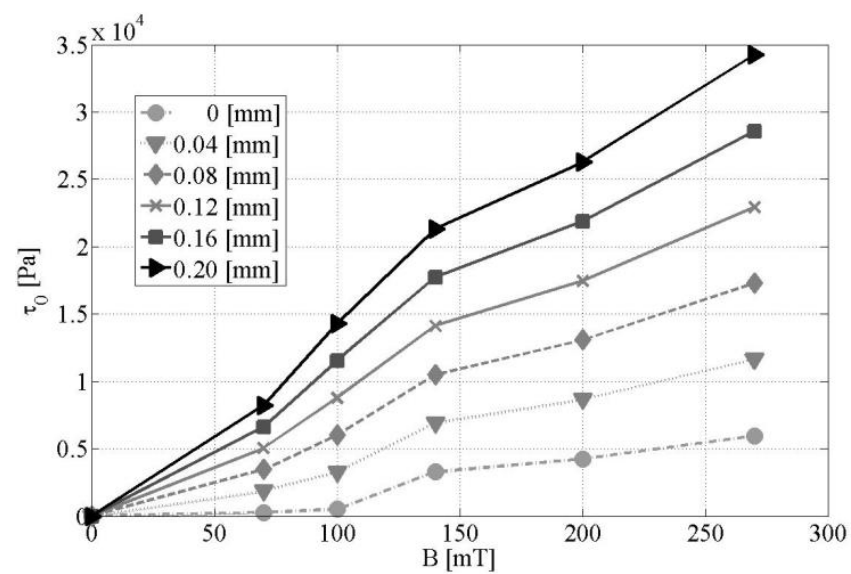

Fig. 7.Yield stress To vs magnetic flux density $B$ for various plate positions $\Delta \mathrm{h}$

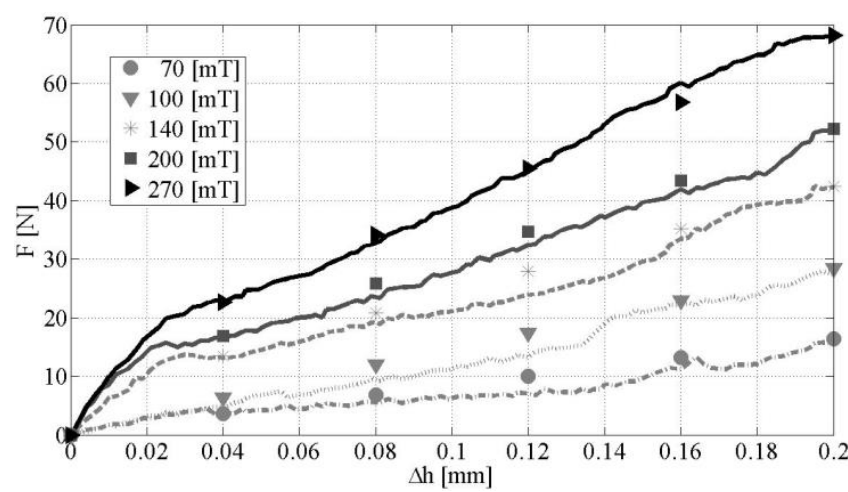

Fig. 8. Squeezing force $F$ vs plate position $\Delta h$ for variousmagneticflux density $B$

The further numerical procedures were applied to ensure a better agreement between the proposed model and experimental data (the determination coefficient being $R^{2}=0.95 \div 0.99$ ). This coefficient is one of the basic measures of the goodness of fit, widely employed in statistics. It is assumed that in the case considered here the yield 
stress of the MR fluid is a function of magnetic flux density and of the plate position $\Delta \mathrm{h}$. The plot of the yield stress in the function of magnetic flux density and the plate position is shown in Fig. 7.

This dependence was then explored by Bingham model simulations. Comparison of CFD calculation data with experimental results is shown in Fig.8. The numerical simulation data are indicated with markers. It is apparent that in the analyzed cases the numerical model well captures the squeezing force variability.

\section{SUMMARY}

This study explores the CFD model of a MR fluid, based on the Bingham model whereby the yield stress is dependent on the height of the fluid gap and flux density in the gap. This model may be useful in design of semi-active and passive devices containing MR fluid, which are being investigated by the authors under the current research project.

Experiments results were obtained for a commercially available MR fluid MRF-122EG (manufactured by Lord Corporation), operated in squeeze mode. The relationship was established between the yield stress and the position of the plate. The proposed viscosity model agrees well with experimental data.

The analysis of MR fluid's behavior in squeeze mode is a major challenge for researchers. In the next step the proposed model should be verified under different excitations applied to the plate and for higher values of the magnetic flux density, taking into account other types of fluids and the real MR devices operated in squeeze mode. The current version of the CFD model fails to take into account the extension of fluids. Preliminary results reveal different values of yield stress when squeezed and when under tension.

\section{REFERENCES}

1. Farjoud A., Cavey R., Ahmadian M., Craft M. (2009), Magnetorheological fluid behavior in squeeze mode, Smart Materials and Structures, 18(9), 1-7.

2. Farjoud A., Craft M., Burke W., Ahmadian M. (2011), Experimental investigation of MR squeeze mounts. Journal of Intelligent Material Systems and Structures, 22:1645-1652.

3. Gavin HP. (2001), Mulit-duct ER dampers, Journal of Intelligent Material Systems and Structures, 12(5), 353-360.

4. Horak W. (2013), Theoretical and experimental analysis of magnetorheological fluids in squeeze mode, PhD Thesis, AGH University of Science and Technology, Krakow.

5. Mazlan S. (2008), The behaviour of magnetorheological fluids in squeeze mode, PhD Thesis, Dublin City University.

6. Rosensweig R.E. (1985), Ferrohydrodynamics, Cambridge University Press, Cambridge.

7. Salwiński J. Horak W. (2012), Measurement of normal force in magnetorheological and ferrofluid lubricated bearings, Key Engineering Material, vol. 490, 25-32.

8. Salwiński J., Horak W., Szczęch M. (2013), Experimental apparatus for examination of magnetic fluid lubricated thrust bearing, XXVI Symposium Fundamentals of Machine Design, Szczyrk, Poland.

9. Sapiński B., Horak W., Szczęch M. (1-4.06.2013), Investigation of MR fluids in the oscillatory squezee mode, VII -th International Symposium on Mechanics materials and Structures, Augustow, Poland.

10. Wang H., Zhang B. J., Liu X. Z., Luo D. Z., Zhong S. B. (2011), Compression resistance of magnetorheological fluid, Advanced Materials Research, 143-144, 624-628.

11. ANSYS Corporation, http://www.ansys.com

12. LORD Corporation, http://www.lord.com

13. Anton-Paar Corporation, http://www.anton-paar.com

14. Labview Corporation, www.ni.com

This research is supported by the National Centre for Research and Development under grant No. PBS 1/A6/3/2012. 